PROCEEDINGS OF THE

AMERICAN MATHEMATICAL SOCIETY

Volume 130, Number 10, Pages 2921-2930

S 0002-9939(02)06416-X

Article electronically published on May 8, 2002

\title{
ON WAVELETS INTERPOLATED FROM A PAIR OF WAVELET SETS
}

\author{
ZIEMOWIT RZESZOTNIK AND DARRIN SPEEGLE
}

(Communicated by David R. Larson)

\begin{abstract}
We show that any wavelet, with the support of its Fourier transform small enough, can be interpolated from a pair of wavelet sets. In particular, the support of the Fourier transform of such wavelets must contain a wavelet set, answering a special case of an open problem of Larson. The interpolation procedure, which was introduced by X. Dai and D. Larson, allows us also to prove the extension property.
\end{abstract}

\section{INTRODUCTION}

The $L^{2}$ theory of orthonormal wavelets is not fully developed yet. There are three ways of understanding the whole set of wavelets in $L^{2}(\mathbb{R})$. The first one relies on characterization equations introduced by Y. Meyer (see [Le], [BSW], Gr, [HW]) or those conjectured by G. Weiss (see $\mathrm{B}, \mathrm{WW}]$ ). The second one is based on the idea of a generalized multiresolution analysis introduced by L. Baggett, H. Medina and K. Merrill in [BMM]. The final one, described by X. Dai and D. Larson in DL, approaches wavelets from the operator theory point of view by treating them as wandering vectors. One of the advantages of this approach is the interpolation procedure, which allows a construction of "new" wavelets from "old" wavelets. The procedure is particularly simple in the case when the "old" wavelets are of the MSF type. As a result, these elementary MSF wavelets can be viewed as "building blocks" of a huge class of wavelets. Even the situation when we use only two wavelet sets to construct new wavelets is very interesting. [DL] contains a detailed study of this basic interpolation. For example, it is shown that Meyer's original construction of wavelets follows naturally from this point of view. The construction of Meyer was based on the characterization equations mentioned above. In this paper we establish a further connection between these equations and wavelets interpolated from a pair of wavelet sets. To be more precise, we use the equations to prove that if the support of $\hat{\psi}$ is "small", then the wavelet $\psi$ can be interpolated from a pair of wavelet sets. To exhibit the usefulness of this basic interpolation, we apply it to prove that the local behavior of the Fourier transform $\hat{\psi}$ of a wavelet $\psi$ is as arbitrary as possible at any point on the real line, except the origin. This is closely related to [BGRW], where it was shown that the origin is a point with a special meaning for $\hat{\psi}$.

Received by the editors September 19, 2000 and, in revised form, March 22, 2001.

2000 Mathematics Subject Classification. Primary 42C40.

Key words and phrases. Orthonormal wavelets, MSF wavelets, interpolated wavelets.

(C)2002 American Mathematical Society 


\section{INTERPOLATION PAIRS OF WAVELET SETS}

Following DL, we shall define an interpolation pair of wavelet sets. Let $E$ and $F$ be wavelet sets. Since both these sets partition $\mathbb{R}$ under translations by $2 k \pi$, $k \in \mathbb{Z}$, we can consider a natural mapping $\sigma: E \rightarrow F$ such that $\sigma(\xi)=\xi+2 k \pi$, where $k \in \mathbb{Z}$. The interpolation map $\sigma_{E}^{F}$ is the unique extension of $\sigma$ to $\mathbb{R}$ such that

$$
\sigma_{E}^{F}(\xi)=2^{j} \sigma_{E}^{F}\left(2^{-j} \xi\right) \quad \text { for } \quad \xi \in 2^{j} E, \quad j \in \mathbb{Z} .
$$

That the extension exists and is unique follows from the fact that both $E$ and $F$ partition $\mathbb{R}$ under dilations by $2^{j}, j \in \mathbb{Z}$. The ordered pair $(E, F)$ is called an interpolation pair if $\left(\sigma_{E}^{F}\right)^{2}$ is equal to the identity. If that is the case, then every function $\psi$ given by

$$
\hat{\psi}=h_{1} \mathbf{1}_{E}+h_{2} \mathbf{1}_{F},
$$

where $h_{1}$ and $h_{2}$ are 2 -dilation-periodic, is an orthonormal wavelet 11 provided that the matrix

$$
\left[\begin{array}{cc}
h_{1} & h_{2} \\
h_{2} \circ \sigma_{E}^{F} & h_{1} \circ \sigma_{E}^{F}
\end{array}\right]
$$

is unitary a.e. on $E$. We refer to such wavelets as wavelets interpolated from a pair $(E, F)$. In this paper, an interpolation pair will be called non-trivial if $E \neq F$. We note here that the trivial case corresponds to MSF wavelets.

The question we are concerned about is: "How big is the class of wavelets interpolated from a pair of wavelet sets?" Although this question is pretty vague, we shall try to give a precise answer to it.

In [La] it is shown that if $(E, F)$ is an interpolation pair and $\psi$ is a wavelet such that the support of $\hat{\psi}$ is contained in $E \cup F$, then $\psi$ can be interpolated from the pair $(E, F)$. Therefore, at least in the case of interpolation pairs, it is enough to concentrate on the support of $\hat{\psi}$ to find out if a given wavelet $\psi$ comes from an interpolation; however, left open is the question of how to know whether the support of $\hat{\psi}$ is contained in the union of an interpolation pair of wavelet sets. This motivates the following approach. Let $\psi \in L^{2}(\mathbb{R})$ be a wavelet. Denote the support of $\hat{\psi}$ by $K$ and define two maps

$$
T_{K}(\xi)=\sum_{k \in \mathbb{Z}} \mathbf{1}_{K}(\xi+2 k \pi)
$$

and

$$
D_{K}(\xi)=\sum_{j \in \mathbb{Z}} \mathbf{1}_{K}\left(2^{j} \xi\right),
$$

where $\xi \in \mathbb{R}$. The maps are defined a.e., and the supremum norms $\left\|T_{K}\right\|_{\infty},\left\|D_{K}\right\|_{\infty}$ determine the "size" of the set $K$. To see how this notion operates let us recall the Meyer-Weiss equations characterizing wavelets (see [HKLS]):

$$
\sum_{k \in \mathbb{Z}}|\hat{\psi}(\xi+2 k \pi)|^{2}=1,
$$

\footnotetext{
${ }^{1}$ We use the Fourier transform defined by

$$
\hat{\psi}(\xi)=\int_{\mathbb{R}} \psi(x) e^{-i x \xi} d x .
$$
}




$$
\begin{gathered}
\sum_{k \in \mathbb{Z}} \hat{\psi}(\xi+2 k \pi) \overline{\hat{\psi}\left(2^{j}(\xi+2 k \pi)\right)}=0 \quad \text { for } \quad j \geq 1, \\
\sum_{j \in \mathbb{Z}}\left|\hat{\psi}\left(2^{j} \xi\right)\right|^{2}=1, \\
t_{q}(\xi):=\sum_{j=0}^{\infty} \hat{\psi}\left(2^{j} \xi\right) \overline{\hat{\psi}\left(2^{j}(\xi+2 q \pi)\right)}=0, \quad \text { for } \quad q \in 2 \mathbb{Z}+1 .
\end{gathered}
$$

A fundamental result in the $L^{2}$ theory of wavelets is that a function $\psi \in L^{2}$ is a wavelet if and only if $\|\psi\|_{2}=1$ and equations (2.5) and (2.6) hold. Moreover, in this case, (2.3) and (2.4) also hold. These facts were proven by various authors in different generalities; see [HW] for details.

From equations (2.3) and (2.5) it follows that the case $\left\|T_{K}\right\|_{\infty}=\left\|D_{K}\right\|_{\infty}=1$ gives us the most elementary wavelets, that is, MSF wavelets (even if only one of the norms is equal to 1 , then $\psi$ must be an MSF wavelet). We will prove that the second simplest case (that is, $\left\|T_{K}\right\|_{\infty}=\left\|D_{K}\right\|_{\infty}=2$ ) corresponds exactly to wavelets interpolated from a non-trivial pair of wavelet sets. Of course, in light of La], we can achieve this by focusing on the support of the Fourier transform of a wavelet.

Theorem 2.1. Let $\psi \in L^{2}(\mathbb{R})$ be an orthonormal wavelet, and let $K$ denote the support of $\hat{\psi}$. There exists a non-trivial interpolation pair $(E, F)$ such that $K=$ $E \cup F$ if and only if $\left\|T_{K}\right\|_{\infty}=\left\|D_{K}\right\|_{\infty}=2$.

Proof. Only one implication of the above statement requires a proof (the other one follows immediately from the fact that $E$ and $F$ are wavelet sets and $E \neq F$ ). The real task is to decompose $K$ into the union of two wavelet sets, provided that $K$ is the support of the Fourier transform of a wavelet $\psi$ and $T_{K}$ and $D_{K}$ are bounded by 2 (proving that these wavelet sets will form an interpolation pair will not be difficult).

The proof consists of several easily-justified claims.

Claim A. $T_{K}(\xi)=D_{K}(\xi)$ for a.e. $\xi \in K$.

Proof of Claim A. To prove Claim A we observe that $T_{K}$ and $D_{K}$ can only attain values 1 and 2 on $\mathbb{R}$ (value 0 is restricted because of (2.3) and (2.5)). From equation (2.3) it follows that $\{\xi \in \mathbb{R}:|\hat{\psi}(\xi)|=1\}=\left\{\xi \in K: T_{K}(\xi)=1\right\}$. Similarly, equation (2.5) asserts that $\{\xi \in \mathbb{R}:|\hat{\psi}(\xi)|=1\}=\left\{\xi \in K: D_{K}(\xi)=1\right\}$. Thus, Claim A is true.

Let $R:=\left\{\xi \in K: T_{K}(\xi)=D_{K}(\xi)=1\right\}$ (we know already that $R=\{\xi \in$ $\mathbb{R}:|\hat{\psi}(\xi)|=1\})$. From Claim A it follows that if $\xi \in K \backslash R$, then there is a unique integer $k \neq 0$ such that $\xi+2 k \pi \in K \backslash R$, and also a unique integer $j \neq 0$ such that $2^{j} \xi \in K \backslash R$. Thus, we can define a map $\tau_{K}: K \backslash R \rightarrow K \backslash R$ by setting

$$
\tau_{K}(\xi)=\xi+2 k \pi,
$$

where $k \in \mathbb{Z} \backslash\{0\}$ ( $k$ depends on $\xi$ ) and a map $d_{K}: K \backslash R \rightarrow K \backslash R$ by setting

$$
d_{K}(\xi)=2^{j} \xi
$$

where $j \in \mathbb{Z} \backslash\{0\}(j$ depends on $\xi)$. 
Claim B. $\tau_{K}$ and $d_{K}$ commute.

Proof of Claim B. We prove Claim B by applying equation (2.6). Let $\xi \in K \backslash R$, $\tau_{K}(\xi)=\xi+2 k \pi$ and $d_{K}(\xi)=2^{j} \xi$. Clearly, $k=2^{n} q$ for some $n \in \mathbb{N} \cup\{0\}$ and $q \in 2 \mathbb{Z}+1$. Since, by $(2.6), t_{q}\left(2^{-n} \xi\right)=0$, we have

$$
\sum_{l=0}^{\infty} \hat{\psi}\left(2^{l-n} \xi\right) \overline{\hat{\psi}\left(2^{l-n}(\xi+2 k \pi)\right)}=0 .
$$

It is easy to see that the above sum contains a non-zero term. Indeed, the term corresponding to $l=n$ is simply $\hat{\psi}(\xi) \overline{\hat{\psi}(\xi+2 k \pi)}$, which cannot be zero, since both $\xi$ and $\xi+2 k \pi$ are in $K$. Thus, to make the whole sum equal to 0 we need to have at least one more non-zero term. On the other hand, since $\xi \in K$ and $D_{K} \leq 2$, we conclude that we can have at most one more non-zero term, the one corresponding to $l=j+n$. Therefore, $(2.7)$ reduces to

$$
\hat{\psi}(\xi) \overline{\hat{\psi}(\xi+2 k \pi)}+\hat{\psi}\left(2^{j} \xi\right) \overline{\hat{\psi}\left(2^{j}(\xi+2 k \pi)\right)}=0 .
$$

The above allows us to draw two conclusions: $2^{j}(\xi+2 k \pi)$ belongs to $K \backslash R$ and $2^{j} k=2^{l} q$ is a non-zero integer. Thus, we have

$$
d_{K} \circ \tau_{K}(\xi)=d_{K}(\xi+2 k \pi)=2^{j}(\xi+2 k \pi)=2^{j} \xi+2^{l} q \pi=\tau_{K}\left(2^{j} \xi\right)=\tau_{K} \circ d_{K}(\xi),
$$

which concludes the proof of the claim.

The fact that the maps $\tau_{K}$ and $d_{K}$ commute can be stated as: "the dilation brother of my translation brother is the translation brother of my dilation brother" and represented by the following diagram:

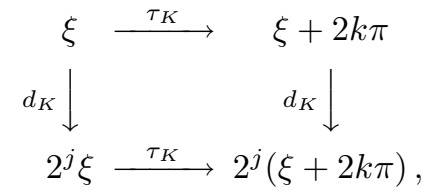

where $2^{j} k \in \mathbb{Z} \backslash\{0\}$. The above diagram, or rather its simplicity, is the main reason why we can decompose $K$ into a union of two wavelet sets. Of course, the set $R$ must be a part of these wavelet sets; thus we only need to investigate the remaining set $K \backslash R$. is,

For every $j, k \in \mathbb{Z} \backslash\{0\}$ we define $K_{j, k}:=\left\{\xi \in K: \xi+2 k \pi \in K, 2^{j} \xi \in K\right\}$; that

$$
K_{j, k}=K \cap(K-2 k \pi) \cap 2^{-j} K .
$$

Since $T_{K}$ and $D_{K}$ are bounded by 2 , it follows that the sets $K_{j, k}$ are mutually disjoint and

$$
\bigcup_{j, k \in \mathbb{Z} \backslash\{0\}} K_{j, k}=K \backslash R .
$$

Claim C. We have the following equalities:

$$
\begin{gathered}
K_{j, k}+2 k \pi=K_{j,-k}, \\
2^{j} K_{j, k}=K_{-j, 2^{j} k}, \\
2^{j}\left(K_{j, k}+2 k \pi\right)=K_{-j,-2^{j} k} .
\end{gathered}
$$


Proof of Claim C. Let $\xi \in K_{j, k}$. By diagram (2.8) we have $2^{j}(\xi+2 k \pi) \in K$. Therefore,

$$
K_{j, k} \subset 2^{-j} K-2 k \pi \text {. }
$$

From (2.9) and (2.11) it follows that

$$
K_{j, k}=K \cap(K-2 k \pi) \cap 2^{-j} K \cap\left(2^{-j} K-2 k \pi\right) .
$$

Thus, since (2.12) holds for $-k$ as well,

$$
K_{j, k}+2 k \pi=(K+2 k \pi) \cap K \cap\left(2^{-j} K+2 k \pi\right) \cap 2^{-j} K=K_{j,-k},
$$

which proves the first equality of the claim. Applying (2.12) in the same manner yields the two remaining equalities.

Claim C allows us to obtain an analogous version of diagram (2.8):

$$
\begin{array}{ccc}
K_{j, k} & \underset{\tau_{K}}{\stackrel{+2 k \pi}{\longrightarrow}} & K_{j,-k} \\
d_{K} \downarrow \cdot 2^{j} & & d_{K} \downarrow \cdot 2^{j} \\
K_{-j, 2^{j} k} & \underset{+2^{j} 2 k \pi}{\stackrel{\tau_{K}}{\longrightarrow}} & K_{-j,-2^{j} k},
\end{array}
$$

where $2^{j} k \in \mathbb{Z} \backslash\{0\}$. Let $A_{j, k}$ denote the union of all the sets in the above diagram, that is,

$$
A_{j, k}:=K_{j, k} \cup K_{j,-k} \cup K_{-j, 2^{j} k} \cup K_{-j,-2^{j} k},
$$

where, to avoid redundancy, we consider only $j, k \in \mathbb{N}$. ( $\mathbb{N}$ does not contain 0.)

Claim D. The sets $A_{j, k}$ are mutually disjoint for all $j, k \in \mathbb{N}$, and

$$
\bigcup_{j, k \in \mathbb{N}} A_{j, k}=K \backslash R
$$

Proof of Claim D. The disjointness follows from the fact that the sets $K_{j, k}$ are mutually disjoint. By (2.10) we have

$$
K \backslash R=\bigcup_{j, k \in \mathbb{N}} K_{j, k} \cup \bigcup_{j, k \in \mathbb{N}} K_{j,-k} \cup \bigcup_{j, k \in \mathbb{N}} K_{-j, k} \cup \bigcup_{j, k \in \mathbb{N}} K_{-j,-k} .
$$

However, if we consider the set $K_{-j, k}$, where $j \in \mathbb{N}$ and $k \in \mathbb{Z} \backslash\{0\}$, then according to diagram (2.13) we must have $2^{-j} k \in \mathbb{Z} \backslash\{0\}$. Therefore, it follows that either $K_{-j, k}$ is empty or $k=2^{j} k^{\prime}$ for some non-zero integer $k^{\prime}$. This observation allows us to reduce the last two unions in (2.14), which leads to

$$
K \backslash R=\bigcup_{j, k \in \mathbb{N}} K_{j, k} \cup \bigcup_{j, k \in \mathbb{N}} K_{j,-k} \cup \bigcup_{j, k^{\prime} \in \mathbb{N}} K_{-j, 2^{j} k^{\prime}} \cup \bigcup_{j, k^{\prime} \in \mathbb{N}} K_{-j,-2^{j} k^{\prime}}=\bigcup_{j, k \in \mathbb{N}} A_{j, k}
$$

and proves our claim.

At this point we are ready to construct the wavelet sets. The intersection with $K \backslash R$ of one of them is obtained by unioning all the terms on the diagonal of diagram (2.13) for $j, k \in \mathbb{N}$, and the intersection of the other one by unioning all the terms on the antidiagonal. Thus, we define

$$
E^{\prime}:=\bigcup_{j, k \in \mathbb{N}}\left(K_{j, k} \cup K_{-j,-2^{j} k}\right), \quad F^{\prime}:=\bigcup_{j, k \in \mathbb{N}}\left(K_{j,-k} \cup K_{-j, 2^{j} k}\right),
$$


and

$$
E:=E^{\prime} \cup R, \quad F:=F^{\prime} \cup R .
$$

Claim D simply assures that

$$
K=E^{\prime} \cup F^{\prime} \cup R
$$

and that the union is disjoint.

Claim E. E and F are wavelet sets.

Proof of Claim E. From diagram (2.13) it follows that both sets are translation and dilation congruent to each other; therefore, it is enough to prove that $E$ is a wavelet set.

From diagram (2.13) it follows that

$$
K \cap \bigcup_{n \in \mathbb{Z} \backslash\{0\}}(E+2 n \pi)=F^{\prime} \quad \text { and } \quad K \cap \bigcup_{n \in \mathbb{Z} \backslash\{0\}} 2^{n} E=F^{\prime} .
$$

Thus, since $E \subset K$, it follows that $\emptyset=E \cap F^{\prime}=E \cap K \cap \bigcup_{n \in \mathbb{Z} \backslash\{0\}}(E+2 n \pi)=$ $E \cap \bigcup_{n \in \mathbb{Z} \backslash\{0\}}(E+2 n \pi)$, and the $2 \pi$-translations of $E$ are disjoint. Similarly, the 2-dilations of $E$ are disjoint. Moreover, by (2.14) and (2.15) we obtain

$$
K \cap \bigcup_{n \in \mathbb{Z}}(E+2 n \pi)=K \quad \text { and } \quad K \cap \bigcup_{n \in \mathbb{Z}} 2^{n} E=K .
$$

Since $T_{K}$ and $D_{K}$ are bounded below by 1 , this proves that $2 \pi$-translations, as well as 2-dilations, of $E$ cover the real line.

Claim F. $(E, F)$ is an interpolation pair.

Proof of Claim F. We need to prove that the interpolation map $\sigma_{E}^{F}$ composed with itself gives us the identity. From (2.1) it follows that it is enough to show that $\left(\sigma_{E}^{F}\right)^{2}(\xi)=\xi$ for a.e. $\xi \in E$. Since $\sigma_{E}^{F}$ restricted to $R$ is the identity, we can focus on the case when $\xi \in E^{\prime}$. Clearly, $\sigma_{E}^{F}(\xi)=\tau_{K}(\xi)$ for $\xi \in E^{\prime}$. Moreover, the same is true if $\xi \in F^{\prime}$. Indeed, from diagram (2.13) it follows that for such $\xi$ 's we have $\sigma_{E}^{F}(\xi)=d_{K} \circ \tau_{K} \circ d_{K}(\xi)=\tau_{K}(\xi)$. Thus, $\sigma_{E}^{F}$ restricted to $K \backslash R$ is equal to $\tau_{K}$, which shows that $\left(\sigma_{E}^{F}\right)^{2}=i d$.

Since we proved that $(E, F)$ is an interpolation pair and, by $(2.14), K=E \cup F$, this concludes the proof of the theorem.

As an immediate consequence of Theorem 2.1 and the remarks preceeding it, we obtain the following characterization.

Corollary 2.2. If $\psi \in L^{2}(\mathbb{R})$ is an orthonormal wavelet and $K$ denotes the support of $\hat{\psi}$, then $\psi$ is interpolated from a pair of wavelet sets $(E, F)$ if and only if $\left\|T_{K}\right\|_{\infty}$ and $\left\|D_{K}\right\|_{\infty}$ are bounded above by 2. Moreover, if this is the case, then either $\left\|T_{K}\right\|_{\infty}=\left\|D_{K}\right\|_{\infty}=2$, in which case $K=E^{\prime} \cup F^{\prime}$, where $\left(E^{\prime}, F^{\prime}\right)$ is a non-trivial interpolation pair, or $\left\|T_{K}\right\|_{\infty}=\left\|D_{K}\right\|_{\infty}=1$, in which case $\psi$ is an MSF wavelet. 


\section{EXTENSION PROPERTY}

The behavior at the origin of the Fourier transform of wavelets was studied in [BGRW]. The starting point of this investigation was the observation that, if $\psi$ is a wavelet and we set $\hat{\psi}(0)=0$, then the origin becomes a Lebesgue point of the function $\hat{\psi}$. It turns out that the origin is the only point on the real line at which the behavior of $\hat{\psi}$ is limited. We shall prove that for any other point $\xi_{0} \in \mathbb{R}$ there exists a neighborhood $U$ such that every measurable function $b$ on $U$ with $|b| \leq 1$ can be extended to the real line in such a way that the extension is the Fourier transform of a wavelet (this result was already announced in BGRW] as Theorem (1.6); the case $|b|=1$ was proven in [IP]). This will be achieved by finding an open interval $U \ni \xi_{0}$, small enough to be a subset of a wavelet set $E$, which will form an interpolation pair with another wavelet set $F$. When this is done, we shall use interpolation based on $(E, F)$ to find a wavelet $\psi$ such that $\left.\hat{\psi}\right|_{U}=b$. This last step of the proof will be familiar to the reader who is accustomed to Meyer's construction of wavelets, which was mentioned before, or its generalizations, presented both in [HWW] and DL].

We start by exhibiting a special class of interpolation pairs which we shall use for our construction; similar classes were considered in [Gu].

Proposition 3.1. Let $T$ and $U$ be measurable subsets of $\mathbb{R}$, and let both $n \in \mathbb{N}$ and $k \in \mathbb{Z}$ be given. If $E:=U \cup\left(2^{-n} U-2 k \pi\right) \cup T$ is a wavelet set and the union is disjoint, then $F:=2^{-n} U \cup\left(U-2^{n} 2 k \pi\right) \cup T$ is a wavelet set and the union is disjoint. Moreover, $(E, F)$ is an interpolation pair.

Proof. If the sets $U, 2^{-n} U-2 k \pi$ and $T$ are pairwise disjoint and their union is a wavelet set, then the sets $2^{-n} U, U-2^{n} 2 k \pi$ and $T$ must be mutually disjoint as well. Therefore, $F$ is translation and dilation congruent to $E$, which shows that $F$ is a wavelet set. We shall prove that $(E, F)$ is an interpolation pair. As we mentioned before, it is enough to show that the interpolation map $\sigma_{E}^{F}$ satisfies $\left(\sigma_{E}^{F}\right)^{2}(\xi)=\xi$ for $\xi \in E$. Computing $\sigma_{E}^{F}$ on $E$ yields

$$
\sigma_{E}^{F}(\xi)= \begin{cases}\xi & \text { for } \xi \in T, \\ \xi-2^{n} 2 k \pi & \text { for } \xi \in U, \\ \xi+2 k \pi & \text { for } \xi \in 2^{-n} U-2 k \pi .\end{cases}
$$

Therefore, on $F, \sigma_{E}^{F}$ has the form

$$
\sigma_{E}^{F}(x)= \begin{cases}\xi & \text { for } \xi \in T, \\ 2^{-n} \sigma_{E}^{F}\left(2^{n} \xi\right)=\xi-2 k \pi & \text { for } \xi \in 2^{-n} U, \\ 2^{n} \sigma_{E}^{F}\left(2^{-n} \xi\right)=\xi+2^{n} 2 k \pi & \text { for } \xi \in U-2^{n} 2 k \pi .\end{cases}
$$

So, it is easily checked that for $\xi \in E,\left(\sigma_{E}^{F}\right)^{2}(\xi)=\xi$.

Although $k$ given in the above proposition can be any integer, we will only need to consider $k=1$ to proceed with our construction. As a technical lemma we shall prove that if $\xi_{0}>0$, then there is a wavelet set $E$, given as in Proposition 3.1, such that $U$ is an open interval containing $\xi_{0}$. We also would like to point out that, according to [BGRW], if $\psi$ is an MRA wavelet and $m$ is an arbitrary integer, then $4 m \pi$ is a Lebesgue point of $\hat{\psi}$, provided $\hat{\psi}(4 m \pi)=0$. This indicates that the case when $\xi_{0}=4 m \pi$ may be harder to consider. Indeed, as we will see, the most general case $\xi_{0} \neq 2 m \pi$ can be treated in a simple way based on the approach offered in 
DLS. The special case $\xi_{0}=2 m \pi$ requires more advanced techniques described in IIP. Both of these methods rely on introducing the maps $\tau: \mathbb{R} \rightarrow[-\pi, \pi)$ defined by $\tau(\xi)=\xi+2 k \pi$ (where $k$ is the unique integer such that $\xi+2 k \pi \in[-\pi, \pi)$ ) and $d: \mathbb{R} \backslash\{0\} \rightarrow[-2 \pi,-\pi) \cup[\pi, 2 \pi)$ defined by $d(\xi)=2^{j} \xi$ (where $j$ is the unique integer such that $2^{j} \xi \in[-2 \pi,-\pi) \cup[\pi, 2 \pi)$ ). Ionascu and Pearcy [IP] proved that a measurable set $V$ is a subset of a wavelet set $E$ if and only if there exist $A \supset V$ and $B \supset V$ such that $\left.\tau\right|_{A},\left.\tau\right|_{B},\left.d\right|_{A}$ and $\left.d\right|_{B}$ are injections, $\tau(A)=[-\pi, \pi)$ and $d(B)=[-2 \pi,-\pi) \cup[\pi, 2 \pi)$. Moreover, in this case, the wavelet set $E$ may be chosen as a subset of $A \cup B$. A sufficient condition for a set $V$ to be contained in a wavelet set was previously noted in [DLS], namely, $\left.\tau\right|_{V}$ and $\left.d\right|_{V}$ are injective, $d(V) \neq[-2 \pi,-\pi) \cup[\pi, 2 \pi)$ and there exists a $\delta>0$ such that $\tau(V) \cap[-\delta, \delta]=\emptyset$. We shall use these two results to prove the technical lemma.

Lemma 3.2. For every $\xi_{0}>0$ there exist an open interval $U \ni \xi_{0}$, a measurable set $T \subset \mathbb{R}$ and some natural $n$ such that $E:=U \cup\left(2^{-n} U-2 \pi\right) \cup T$ is a wavelet set and the union is disjoint.

Proof. Case 1: $\xi_{0} \neq 2 m \pi$ for any $m \in \mathbb{N}$. Let $U$ be an open neighborhood of $\xi_{0}$ such that $2 U \cap U=\emptyset$ and $2 k \pi \notin U$ for any $k \in \mathbb{Z}$. Write $U=\left(2 k \pi+\epsilon_{1}, 2(k+1) \pi-\epsilon_{2}\right)$, where $\epsilon_{1}, \epsilon_{2}>0$ and $k \in \mathbb{Z}$. Pick $n \in \mathbb{N}$ such that $2^{-n} U \subset\left[0, \epsilon_{1}\right] \cap[0, \pi / 2]$ and denote the disjoint union $U \cup\left(2^{-n} U-2 \pi\right)$ by $V$. Notice that $\left.\tau\right|_{V}$ is $1-1,\left.d\right|_{V}$ is $1-1$, $\tau(V) \cap[-\delta, \delta]=\emptyset$ for $\delta=\min \left(2^{-n}\left(2 k \pi+\epsilon_{1}\right), \epsilon_{2}\right)$, and $d(V) \neq[-2 \pi,-\pi) \cup[\pi, 2 \pi)$. Hence, by [DLS], there is a $T \subset \mathbb{R}$ such that $V \cup T$ is a wavelet set, as desired.

Case 2: $\xi_{0}=2 m \pi$ for some $m \in \mathbb{N}$. For $m=1$ we can choose $U=(4 \pi / 3,8 \pi / 3)$, $T=\emptyset$ and $n=1$. For $m \neq 1$, choose $n \in \mathbb{N}$ such that $2^{-n} \xi \in(2 \pi / 3,4 \pi / 3)$, and choose $U$ such that $2^{-n} U \subset(2 \pi / 3,4 \pi / 3)$ and $U \subset(2 m \pi-\pi / 3,2 m \pi+\pi / 3)$. Notice that $\tau\left(2^{-n} U\right) \subset(2 \pi / 3, \pi) \cup[-\pi,-2 \pi / 3)$ and $\tau(U) \subset(-\pi / 3, \pi / 3)$, so, setting $V=U \cup\left(2^{-n} U-2 \pi\right)$, we see that $\left.\tau\right|_{V}$ and $\left.d\right|_{V}$ are 1-1.

We wish to apply the theorem from [IP] which characterizes sets which are subsets of wavelet sets. In order to do so, we must find sets $T_{1}$ and $T_{2}$ (both disjoint from $V$ ) such that the restrictions of $\tau$ and $d$ to $T_{1} \cup V$ and $T_{2} \cup V$ are 1-1, $\tau\left(T_{1} \cup V\right)=[-\pi, \pi)$ and $d\left(T_{2} \cup V\right)=[-2 \pi,-\pi) \cup[\pi, 2 \pi)$.

We simply note that $T_{1}=[2(m-1) \pi+\pi / 3,2 m \pi+\pi / 3) \cap U^{c} \cap\left(2^{-n} U+2(m-1) \pi\right)^{c}$ and $T_{2}=1 / 2\left([2 \pi / 3,4 \pi / 3) \cap\left(2^{-n} U\right)^{c}\right) \cup 1 / 2\left([-4 \pi / 3,-2 \pi / 3) \cap\left(2^{-n} U-2 \pi\right)^{c}\right)$ satisfy the conditions above. Therefore, by Ionascu and Pearcy's characterization, there is a set $T \subset T_{1} \cup T_{2}$ such that the disjoint union $U \cup\left(2^{-n} U-2 \pi\right) \cup T$ is a wavelet set.

From Proposition 3.1 it follows that the set $E=U \cup\left(2^{-n} U-2 \pi\right) \cup T$ from Lemma 3.2 and the set $F=2^{-n} U \cup\left(U-2^{n} 2 \pi\right) \cup T$ form an interpolation pair. The standard interpolation procedure allows us to prove the main result of this section, which generalizes the special case $|b|=1$ proven in [IP].

Theorem 3.3. If $\xi_{0} \neq 0$, then there exists a neighborhood $U$ of $\xi_{0}$ with the property that if $b$ is any measurable function on $U$ with $|b| \leq 1$, then we can find a wavelet $\psi$ such that $\hat{\psi}(\xi)=b(\xi)$ for all $\xi \in U$.

Proof. First we observe that if $\psi$ is a wavelet, then $\hat{\psi}(-\cdot)$ is the Fourier transform of a wavelet (this follows from the Meyer-Weiss equations). Therefore, we can restrict our attention to the case $\xi_{0}>0$.

Let $U, T$ and $n$ be as in Lemma 3.2. We will interpolate from the pair $(E, F)$, where $E=U \cup\left(2^{-n} U-2 \pi\right) \cup T$ and $F=2^{-n} U \cup\left(U-2^{n} 2 \pi\right) \cup T$. In order to do 
so we define $b_{1}, b_{2}, b_{3}$ and $b_{4}$ by

$$
\begin{aligned}
& b_{1}(\xi)=\sqrt{1-\left|b\left(2^{n} \xi\right)\right|^{2}} \text { for } \xi \in 2^{-n} U \text { and } 0 \text { otherwise, } \\
& b_{2}(\xi)=\sqrt{1-b_{1}^{2}(\xi+2 \pi)} \text { for } \xi \in 2^{-n} U-2 \pi \text { and } 0 \text { otherwise, } \\
& b_{3}(\xi)=\sqrt{1-b_{2}^{2}\left(2^{-n} \xi\right)} \text { for } \xi \in U-2^{n} 2 \pi \text { and } 0 \text { otherwise, } \\
& b_{4}(\xi)=1 \text { for } \xi \in T \text { and } 0 \text { otherwise. }
\end{aligned}
$$

A simple calculation shows that if we set $h_{1}(\xi)=e^{i \xi / 2}\left(|b(\xi)|+b_{2}(\xi)+b_{4}(\xi)\right)$ on $E$ and $h_{2}(\xi)=e^{i \xi / 2}\left(b_{1}(\xi)+b_{3}(\xi)\right)$ on $F$ and extend 2-dilation-periodically, then the matrix (2.2) is unitary on $E$ and, therefore, $\psi$ given by $\hat{\psi}=h_{1} \mathbf{1}_{E}+h_{2} \mathbf{1}_{F}$ is a wavelet. To finish the proof we observe that there exists a unimodular function $\nu$ defined on $U$ such that $b(\xi)=\nu(\xi) e^{i \xi / 2}|b(\xi)|$. Moreover, since $U$ is an open interval whose measure does not exceed $2 \pi$, we can extend $\nu$ to a $2 \pi$-periodic unimodular function $\tilde{\nu}$. Then, by the Meyer-Weiss equations, $\tilde{\nu}(\xi) \hat{\psi}(\xi)$ is the Fourier transform of a wavelet and $\tilde{\nu}(\xi) \hat{\psi}(\xi)=b(\xi)$ for all $\xi \in U$, as desired.

We also point out that applying the above theorem to a point $\xi_{0}=4 m \pi$, where $m$ is a non-zero integer, and a bell-shaped smooth function $b$ yields an example of a non-MRA wavelet which behaves nicely on the interval $U$. A similar result is proven in $[\overline{\mathrm{BCM}}]$; the authors became aware of it after submitting this paper.

\section{Conclusion}

We answered the question: "How big is the class of wavelets interpolated from a pair of wavelet sets?" in two ways. The proof of the extension property indicated that, at least locally, every wavelet can be approximated by those wavelets in the frequency domain. The characterization theorem, presented as Corollary 2.2, asserted that a minimal necessary condition on the support of the Fourier transform of an interpolated wavelet is also sufficient to assure that a wavelet comes from interpolation. In this sense the class is as big as possible. The main difficulty in proving this characterization was in finding a wavelet set contained in the support of the Fourier transform of a given wavelet. The problem of doing so in general was already formulated by D. Larson in La], and its solution does not follow immediately from the present $L^{2}$ theory of wavelets.

\section{ACKNOWLEDGMENTS}

We would like to thank Professor Guido Weiss for bringing our attention to the extension property.

\section{REFERENCES}

[B] Bownik, M., On Characterizations of multiwavelets in $L^{2}\left(\mathbb{R}^{n}\right)$, Proc. Amer. Math. Soc. 129 (2001), 3263-3274. CMP 2001:16

[BCM] Baggett, L., Courter, J. and Merrill, K., The construction of wavelets from generalized conjugate mirror filters in $L^{2}\left(\mathbb{R}^{n}\right)$, preprint.

[BGRW] Brandolini L., Garrigós G., Rzeszotnik Z. and Weiss G., The behaviour at the origin of a class of band-limited wavelets, Contemporary Mathematics 247 (1999), 75-91. MR 2001b: 42045

[BMM] Baggett, L.W., Medina, H.A. and Merrill, K.D., Generalized multiresolution analyses, and a construction procedure for all wavelet sets in $\mathbb{R}^{n}$, J. Fourier. Anal. Appl. 5 (no.6) (1999), 563-573. MR 2001f: 42055 
[BSW] Bonami, A., Soria, F. and Weiss, G., Band-limited wavelets, Jour. of Geom. Anal. 3 no.6 (1993), 543-578. MR 94k:42046

[DL] Dai, X. and Larson, D.R., Wandering vectors for unitary systems and orthogonal wavelets, Mem. Amer. Math. Soc. 134 (no. 640) (1998). MR 98m:47067

[DLS] Dai, X., Larson, D.R. and Speegle, D., Wavelet sets in $\mathbb{R}^{n}$, II, Contemp. Math. 216 (1998), 15-40. MR 99d:42054

[Gr] Gripenberg, G., A necessary and sufficient condition for the existence of a father wavelet, Studia Math. 114 (1995), 207-226. MR 96d:42049

[Gu Gu, Q., On interpolation families of wavelet sets, Proc. Amer. Math. Soc. 128 (2000), 2973-2979. MR 2000m:42026

[HKLS] Ha, Y., Kang, H., Lee, J. and Seo, J., Unimodular wavelets for $L^{2}$ and the Hardy space $H^{2}$, Michigan Math. J. 41 (1994), 345-361. MR 95g:42050

[HW] Hernández, E. and Weiss, G., A first course on wavelets, Studies in Advanced Mathematics, CRC Press, Boca Raton, FL, 1996. MR 97i:42015

[HWW] Hernández, E., Wang, X. and Weiss, G., Smoothing minimally supported frequency wavelets. I, J. Fourier Anal. Appl. 2 no. 4 (1996), 329-340. MR 97h:42015

[IP] Ionascu, E.J. and Pearcy, C.M., On subwavelet sets, Proc. Amer. Math. Soc. 126 (1998), 3549-3552. MR 99b:42040

[La] Larson, D.R., Von Neumann algebras and wavelets, Operator algebras and applications (Samos, 1996), 267-312, NATO Adv. Sci. Inst. Ser. C Math. Phys. Sci., 495, Kluwer Acad. Publ., Dordrecht, 1997. MR 98g:46091

[Le] Lemarié-Rieusset, P.G., Ondolettes à localisation exponentielle, J. Math. Pure et Appl 67 (1988), 227-236. MR 89m:42024

[WW] Weiss G. and Wilson E.N., The Mathematical Theory of Wavelets, preprint.

Institute of Mathematics, University of Wroclaw, Pl Grunwaldzki 2/4, 50-384 WroClaw, Poland

E-mail address: zioma@math.uni.wroc.pl

Department of Mathematics \& Computer Science, Saint Louis University, St. Louis, Missouri 63103

E-mail address: speegled@slu.edu 